LA-UR-01-4478 CEC/ICMC 2001, Madison, WI, July 16-20, 2001 Paper I-15B-03

Accepted for publication in Advances in Cryogenics, vols. 47-48, eds. U.

Balachandran et al., American Institute of Physics, March 2002.

\title{
ENHANCED CRITICAL CURRENTS IN Bi-2223/Ag SHEATHED TAPES BY HIGH ENERGY PROTON IRRADIATION
}

\author{
J. O. Willis ${ }^{1}$, J. Y. Coulter ${ }^{1}$, M. P. Maley ${ }^{1}$, J. L. Ullmann ${ }^{1}$, \\ S. Fleshler ${ }^{2}$, and G. N. Riley, Jr. ${ }^{2}$ \\ ${ }^{1}$ Superconductivity Technology Center, MS-K763, MST-STC \\ Los Alamos National Laboratory, Los Alamos, NM 87545 USA \\ ${ }^{2}$ American Superconductor Corporation \\ Two Technology Drive, Westborough, MA 01581 USA
}

\begin{abstract}
We irradiated $\mathrm{Bi}-2223 / \mathrm{Ag}$ sheathed tapes with high-energy protons, causing fission reactions with $\mathrm{Bi}$ and $\mathrm{Pb}$ nuclei and generating in situ splayed columnar defects in the superconductor core of the tapes. We find that the best $75 \mathrm{~K}$ critical-current density $\mathrm{J}_{\mathrm{c}}$ performance in an applied magnetic field B perpendicular to the tape plane is achieved at a matching field, for which the flux-quantum density equals the columnar-defect density, of about $1 \mathrm{~T}$. At this defect density, the $\mathrm{J}_{\mathrm{c}}$ at $75 \mathrm{~K}$ and $1 \mathrm{~T}$ perpendicular to the tape plane is almost 100 times larger than that for an unirradiated tape. For higher proton doses, there is increasingly greater grain-boundary damage, lowering the self-field $\mathrm{J}_{\mathrm{c}}$ and ultimately degrading the in-field performance. However, much of this damage can be repaired with a short anneal at $400^{\circ} \mathrm{C}$. Decreasing the incident proton energy from 800 to $500 \mathrm{MeV}$ has little effect on the enhanced $\mathrm{J}_{\mathrm{c}}$ performance and has beneficial side effects. Irradiation of a stack of tapes demonstrates the high penetration power and uniform generation of columnar defects throughout a substantial volume of material. The application of this technique to improved device performance will be discussed.
\end{abstract}

\section{INTRODUCTION}

High temperature superconductor (HTS) tapes are being considered for the current carrying component of power utility industry devices, such as cables, transformers, fault current limiters, motors, and generators. Such devices require the HTS tapes to be available in long lengths $(>1000 \mathrm{~m})$, to be flexible and mechanically strong for handling and winding, and to have a large critical current density $J_{c}$ in the ambient magnetic field of less than $0.1 \mathrm{~T}$ for cables and up to $\sim 5 \mathrm{~T}$ for motors and generators. Bi-2223/Ag sheathed tapes are the only HTS materials presently available for such applications at liquid nitrogen temperatures. However, at these temperatures thermally activated magnetic flux motion is severe in the bismuth-based superconductors, which are electronically highly anisotropic and nearly two dimensional, and this tends to reduce $\mathrm{J}_{\mathrm{c}}$ in the presence of a magnetic field. As a result, the useful operating range of $\mathrm{Bi}-2223$ at $77 \mathrm{~K}$ is less than $0.5 \mathrm{~T}$. 
The introduction of columnar defects by high-energy, heavy ion produces large enhancements in the magnetization $J_{c}$ and raises the irreversibility line in single crystal Bi-2212 [1]. Both of these factors increase the useful operating field at high temperatures. However, the heavy ions penetrate only 20-30 $\mu \mathrm{m}$ into the HTS material, a distance insufficient to penetrate a typical OPIT tape of thickness $100-200 \mu \mathrm{m}$, and thus are impractical for generating pinning centers throughout the volume of manufacturable conductor geometries.

Another technique, using high-energy protons, has been developed that overcomes these limitations. When a proton with energy greater than $\sim 100 \mathrm{MeV}(0.1 \mathrm{GeV})$ bombards a $\mathrm{Bi}-2223 / \mathrm{Ag}$ tape, a small fraction of the protons interact with the heavy nuclei in the tape $(\mathrm{Bi}$ and $\mathrm{Pb}$ ) producing nuclear fission reactions. In fact, most protons pass through the tape with no interaction, since the mean free path in matter for $800 \mathrm{MeV}$ protons is $\sim 50 \mathrm{~cm}$. The two roughly equal mass fission fragments recoil through the lattice with a combined energy of $\sim 200 \mathrm{MeV}$ creating a columnar damage track $\sim 6 \mu \mathrm{m}$ long and 5-10 $\mathrm{nm}$ in diameter. The tracks are random in orientation with respect to the incident proton beam and are called splayed columnar defects. These have been predicted theoretically [2] to be more efficient in pinning magnetic flux (and thus enhancing $\mathrm{J}_{\mathrm{c}}$ ) in two-dimensional HTS systems, such as $\mathrm{Bi}-2212$ or $\mathrm{Bi}-2223$, than are parallel columnar defects produced by heavy ion irradiation.

The original work had investigated the effects of proton induced splayed columnar defects in Bi-2212 single crystals and $\mathrm{Bi}-2212 / \mathrm{Ag}$ surface coated tapes [3]. This was followed shortly by an investigation of proton-irradiated $\mathrm{Bi}-2223 / \mathrm{Ag}$ sheathed tapes by the Los Alamos group [4-6]. For magnetic fields up to $\sim 1 \mathrm{~T}$ at $75 \mathrm{~K}$, large enhancements were seen in the critical current for the magnetic field oriented along the normal to the tape plane. This is roughly parallel to the c axis for well-aligned grains in the tape. For this direction of the magnetic field, the pinning is rather weak in unirradiated Bi-2223. For magnetic field parallel to the tape plane, on the other hand, the intrinsic pinning of flux vortices by the crystallographic $a b$ planes is rather strong, and proton irradiation produced insignificant critical current enhancements.

Another technique to produce in situ splayed columnar defects has also been developed [7]. There, the Bi-2223 powder is doped with ${ }^{235} \mathrm{U}-\mathrm{O}$. After the Bi-2223/Ag tape has been thermomechanically processed, the tape is irradiated with thermal neutrons. The ${ }^{235} \mathrm{U}$ has a large fission cross section for thermal neutrons, and the energetic fission products create the splayed columnar defects.

Below are described the experimental techniques for tape fabrication, irradiation, dosimetry and superconductor characterization. This is followed by a discussion of the critical current $I_{c}$ performance as a function of proton dose, post irradiation annealing, proton energy dependence, and activation analysis. Finally, the results are compared with those of others and some conclusions are drawn.

\section{EXPERIMENTAL}

\section{Tape Fabrication}

Multi (85)-filament Bi-2223/Ag tape samples were manufactured by American Superconductor Corporation as one of their early commercial products employing a standard OPIT process [8]. Tapes were prepared by packing the powder into cylindrical silver billets, which were deformed into tape using standard deformation techniques, including wire drawing to a hexagonal shape, repacking an 85 filament bundle into a silver tube, wire drawing, and rolling. The samples were given multiple heat treatments with intermediate deformations. The 85 filament tapes have final dimensions of $0.25 \times 2.5 \mathrm{~mm}$ with filament dimensions of $15 \mu \mathrm{m}$ thick and $120 \mu \mathrm{m}$ wide and a $28 \%$ fill factor. These tapes were of an older generation to allow comparison with results of earlier experiments [4-5]. The critical current $\mathrm{I}_{\mathrm{c}}$ of the tape stock is about $30 \mathrm{~A}$ at $77 \mathrm{~K}, 0 \mathrm{~T}$; the $\mathrm{J}_{\mathrm{c}}$ and $\mathrm{J}_{\mathrm{e}}$ are 20 and $\sim 4.5 \mathrm{kA} / \mathrm{cm}^{2}$, respectively, under the same conditions. 
Irradiations and Dosimetry

The irradiations took place at the WNR facility of the Los Alamos Neutron Science Center. The beam has an axially symmetric Gaussian distribution in the density of incident protons with a full width at half maximum of $1.2 \mathrm{~cm}$. Several techniques were used to measure the proton dose received by each sample. A counter measured the number of micropulses, at $\sim 10^{8}$ protons per micropulse, of beam that each sample received. This is the primary measure of relative dose. A current monitor, which is simply a pickup coil surrounding the beam line and in which a voltage is induced when a (proton) current flows through it, and activation of thin Al foil disks $6.3 \mathrm{~mm}$ in diam placed directly behind the samples were also used to confirm the proton dose.

The number of fissions per unit volume per proton was determined as the product of the proton fluence, the density of $\mathrm{Bi}$ nuclei in $\mathrm{Bi}-2223\left(7.33 \times 10^{21}\right.$ nuclei $\left./ \mathrm{cm}^{3}\right)$, and the $\mathrm{Bi}$ fission cross section value of $155 \mathrm{mb}$ at 800 or $500 \mathrm{MeV}$ interpolated from literature values [9]. Assuming an average track length of $6 \mu \mathrm{m}$ [3] for each fission, an areal density of tracks was calculated. The track density was then multiplied by the flux quantum $\phi_{0}(=$ $2.07 \times 10^{-11}$ Tesla- $\mathrm{cm}^{2}$ ), to convert it to an equivalent magnetic field $\mathrm{B}_{\phi}$, at which the vortex density equals the track density. The tapes were irradiated at several fluences from 1 $\mathrm{T}$ to $4 \mathrm{~T}$. To investigate the effect of incident proton energy on the nature of the defects produced and the amount of induced radioactivity, energies of 500 and $800 \mathrm{MeV}$ were selected. Finally, to look at scale-up issues, from 2 to 9 tapes were irradiated at a time, in various configurations, to determine the effect of the beam on multiple layers of tape.

\section{Measurements}

The activation measurements were made with a Eberline Smart Portable Model ESP-1 counter at a distance of $10 \mathrm{~cm}$ from the sample. It was calibrated with a depleted uranium source of a known activity. This type of counter is sensitive to soft (low energy) $\beta$ and $\gamma$ radiation, as well as to harder $\gamma$ radiation.

The $I-V$ characteristics of the samples were measured with the samples immersed in liquid nitrogen to avoid heating at the contacts. The critical current $\mathrm{I}_{\mathrm{c}}$ was determined at an electric field criterion of $1 \mu \mathrm{V} / \mathrm{cm}$. Measurements were made as a function of temperature and magnetic field angle and amplitude.

\section{RESULTS AND DISCUSSION}

\section{Transport Results}

The primary result of the transport critical current $I_{c}$ measurements for a set of tapes irradiated to several defect densities $\mathrm{B}_{\phi}$ from 0 to $\sim 3 \mathrm{~T}$ is shown in FIG 1. For the magnetic field parallel to the tape plane (not shown), there is essentially no enhancement of $I_{c}$. For the magnetic field parallel to the tape normal, the $I_{c}$ values are enhanced up to about $1 \mathrm{~T}$. At higher defect densities, the self-field $\mathrm{I}_{\mathrm{c}}$ is degraded, and at $2 \mathrm{~T}$ and $2.8 \mathrm{~T}$ defect densities clear weak-link like behavior is observed. This suggests significant damage to the grain boundaries. An annealing study discussed next indicates a process to repair some of this damage while maintaining the enhanced field dependence produced by the splayed columnar defects.

\section{Post Irradiation Annealing Study}

As seen earlier in FIG 1, high densities of columnar defects resulted in weak-link behavior. The operating premise for annealing the tapes is that grain boundaries may be more sensitive to damage than the intragranular region, and that damage at the grain boundaries may also be easier to heal. Annealing studies on Bi-2212 crystals, where there 
are no grain boundaries, indicated that annealing temperatures below about $500-600^{\circ} \mathrm{C}$ would not anneal away the beneficial defects within the grain. Three tapes irradiated to nominal defect densities $\mathrm{B}_{\phi}$ of $1 \mathrm{~T}, 1.3 \mathrm{~T}$, and $2.8 \mathrm{~T}$ were annealed at $400^{\circ} \mathrm{C}$ for 2 hours in $10 \% \mathrm{O}_{2} /$ Ar. For the tape irradiated to $1 \mathrm{~T}$, there was no clear evidence of extra weak link damage in the irradiated but unannealed tape; the critical current at zero field was not degraded with proton irradiation at this dose level. What was observed after annealing was a slight decrease in the field performance for B parallel to the tape normal (B\|Normal), although it is still significantly better than that for the unirradiated reference sample.

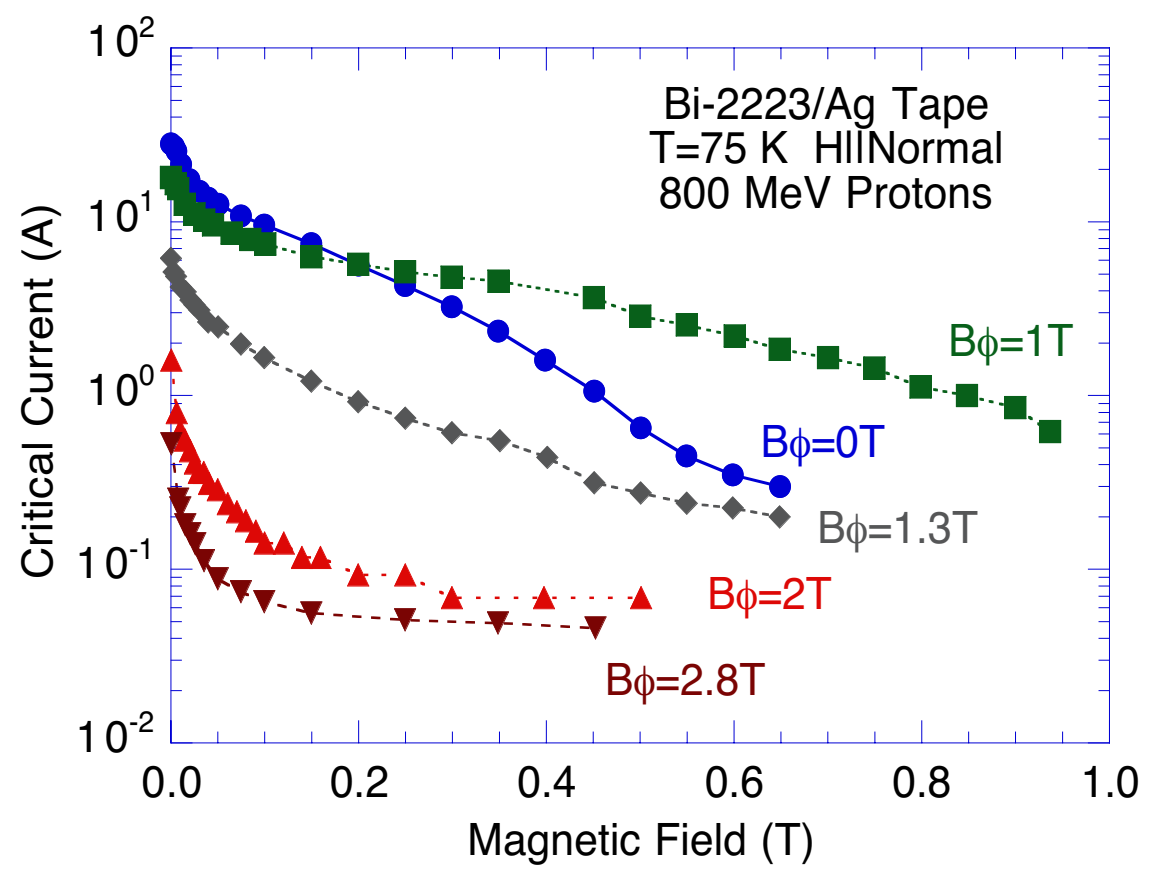

FIGURE 1. Critical current $\mathrm{I}_{\mathrm{c}}$ versus applied magnetic field $\mathrm{H}$ for $\mathrm{Bi}-2223 / \mathrm{Ag}$ tapes irradiated with 800 $\mathrm{MeV}$ protons to defect densities $\mathrm{B}_{\phi}$ from 0 to $\sim 3 \mathrm{~T}$. The data for $\mathrm{H}$ parallel to the tape normal (approximately along the c-axis direction for well aligned grains) is shown. $\mathrm{I}_{\mathrm{c}}$ for $\mathrm{H}$ parallel to the tape plane is unchanged for irradiated tapes until the self-field $I_{c}$ begins to degrade.

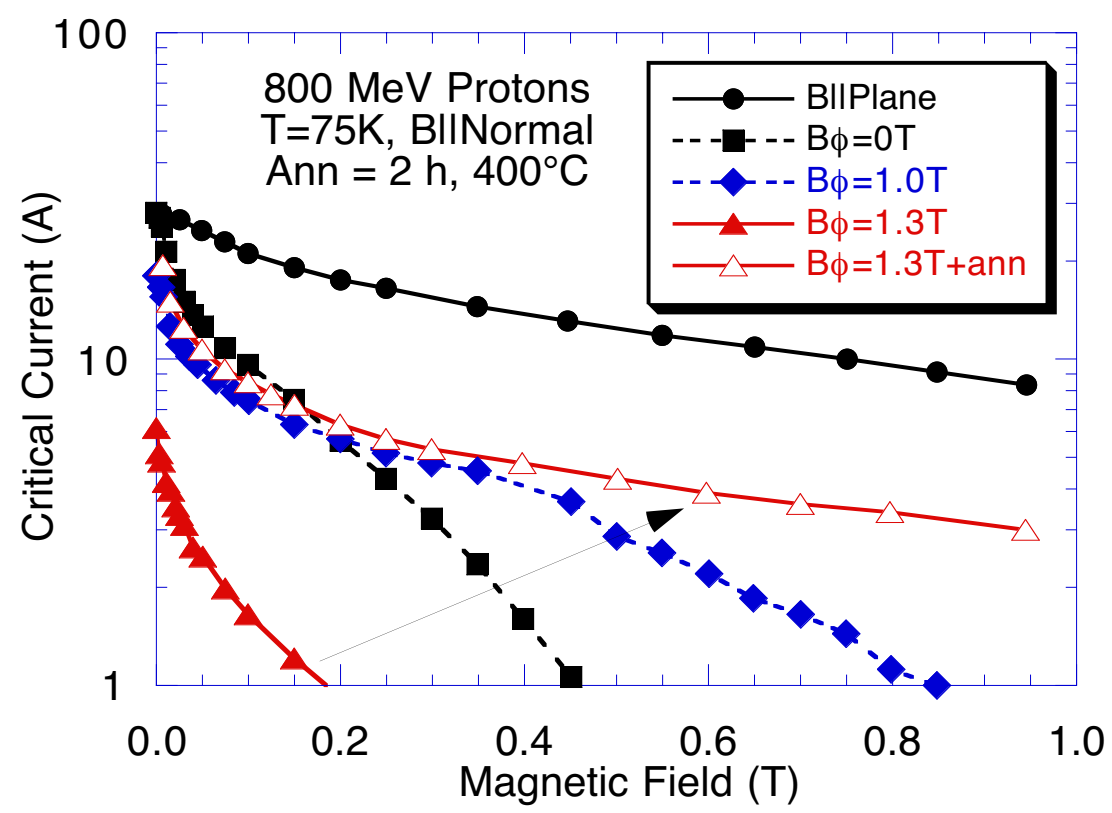

FIGURE 2. Critical current of an unirradiated tape, one irradiated to defect density $\mathrm{B}_{\phi}=1.3 \mathrm{~T}$, and a tape irradiated to $1.3 \mathrm{~T}$ and then annealed at $400^{\circ} \mathrm{C}$ for 2 hours. For reference, the best result obtained at $800 \mathrm{MeV}$ in this study, which is at a defect density $\mathrm{B}_{\phi}$ of $1 \mathrm{~T}$, is also shown. 
The tape irradiated to $\mathrm{B}_{\phi}=2.8 \mathrm{~T}$ showed major degradation in $\mathrm{I}_{\mathrm{c}}$ down to $0.6 \mathrm{~A}$ and characteristic weak link field performance. The $400^{\circ} \mathrm{C}, 2 \mathrm{~h}$ anneal raises $\mathrm{I}_{\mathrm{c}}$ almost a factor of 10 to $5 \mathrm{~A}$, indicating the validity of the original thesis, but not demonstrating the level of recovery desired. The final sample examined is from a set irradiated to $\mathrm{B}_{\phi}=1.3 \mathrm{~T}$; the results are illustrated in FIG 2. The irradiated tape has a degraded $\mathrm{I}_{\mathrm{c}}$ of about $6 \mathrm{~A}$ and shows evidence of weak link behavior in the field dependence. However, after the standard anneal, $\mathrm{I}_{\mathrm{c}}$ at zero field has recovered almost to the unirradiated value, and, instead of being depressed below that of the unirradiated sample, is considerably enhance above it. The I-V curves at low field are complicated by a small linear "foot" for the irradiated samples. The data in FIG 2 were obtained by applying the $1-\mu \mathrm{V} / \mathrm{cm}$ criterion with this foot as the baseline. While this may affect the interpretation of the data at low fields, for fields greater than about $0.5 \mathrm{~T}$, there is a negligible effect, and there is an unequivocal improvement in $\mathrm{I}_{\mathrm{c}}$. In fact, as FIG 2 shows, the combination of $\mathrm{B}_{\phi}=1.3 \mathrm{~T}$ defects plus the standard post anneal results in better performance at $1 \mathrm{~T}$ than does the best result for the same proton energy of $800 \mathrm{MeV}$, which was obtained at $\mathrm{B}_{\phi}=1 \mathrm{~T}$. This annealing result is also comparable to the best result obtained at $500 \mathrm{MeV}$, which was also at $\mathrm{B}_{\phi}=1 \mathrm{~T}$.

To investigate the dependence of the pinning enhancement on the energy of the incident proton beam, two tapes were irradiated to the same defect density $\mathrm{B}_{\phi}$ of $1 \mathrm{~T}$ with $800 \mathrm{MeV}$ and $500 \mathrm{MeV}$ protons, respectively, and the critical current performance was measured at $75 \mathrm{~K}$. Both tapes showed nearly identical magnetic field dependence and showed strong enhancements over the $I_{c}$ for an unirradiated tape for the magnetic field normal to the tape plane for fields greater than about $0.2 \mathrm{~T}$. There was clearly no decrease in the effectiveness of the lower energy protons.

The result of a study of the uniformity of enhancements to a stack of 9 tapes irradiated simultaneously is shown in FIG 3. These tapes were the first, fifth, and ninth in the stack, respectively. The upper curve in the figure shows the result for $\mathrm{H}$ parallel to the tape plane; this is independent of the position of the tape in the stack and identical to the value for the unirradiated reference sample. For $\mathrm{H}$ normal to the tape plane, there is only a small difference with position and only for $\mathrm{H}$ larger than $0.5 \mathrm{~T}$. Because the proton (and therefore defect) density varies spatially on the scale of a few millimeters, slight differences in the placement of voltage contacts may lead to sampling of different average defect densities. At least some of the differences observed in FIG 3 may be related to small differences in the placement of the voltage contacts. Note that these differences are still quiet small when compared to the large enhancement of the critical current relative to the unirradiated sample (lowest curve).

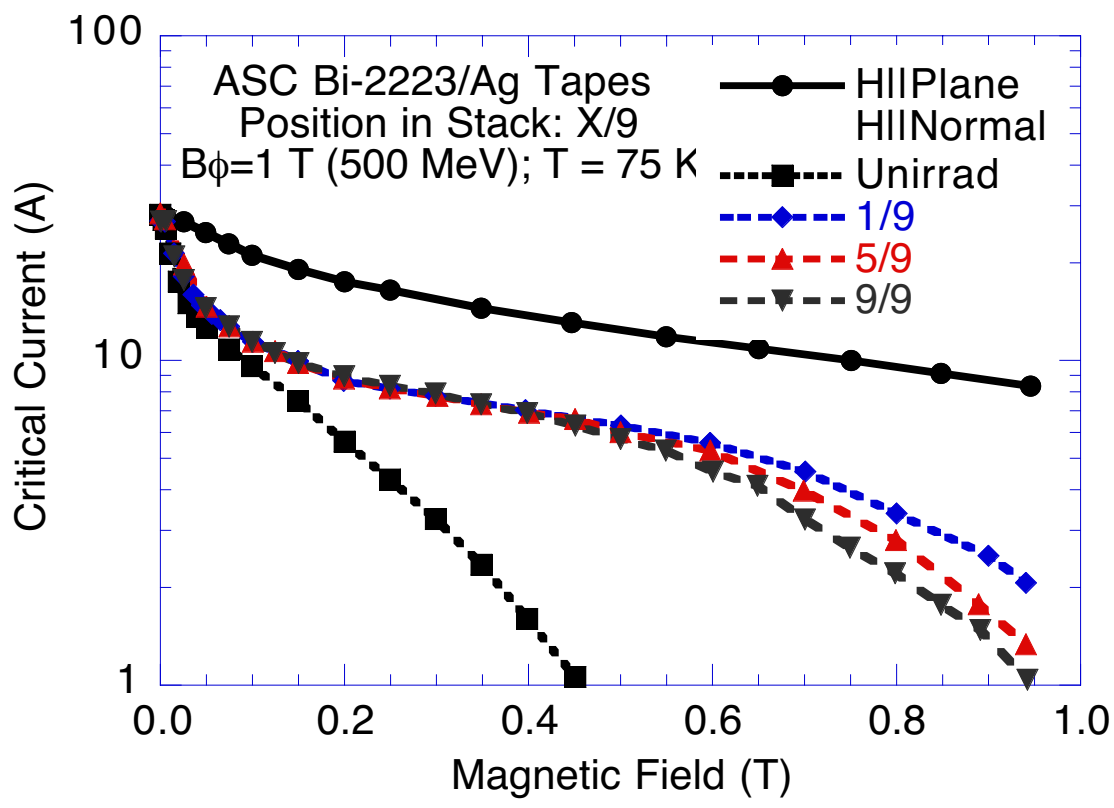

FIGURE 3. Comparison of critical currents at $75 \mathrm{~K}$ for three tapes irradiated simultaneously to a defect dose $\mathrm{B} \phi$ of $1 \mathrm{~T}$. Reference data for an unirradiated tape are also shown. 




FIGURE 4. Comparison of critical current as a function of magnetic field at $75 \mathrm{~K}$ for an unirradiated tape and one irradiated to $\mathrm{a}_{\phi}$ of $1.1 \mathrm{~T}$.

Measurement of the critical current in magnetic fields to $6 \mathrm{~T}$ (FIG 4) shows that for fields above about $1 \mathrm{~T}$, the enhancement in $\mathrm{I}_{\mathrm{c}}$ for $\mathrm{B} \|$ tape normal continues out to at least 2 T. For B ||tape plane, no enhancement is seen at fields below about $1 \mathrm{~T}$, as was noted earlier, but there is a significant enhancement above that field value, e.g., nearly a factor of 10 at $5 \mathrm{~T}$. The Bi-2223 grains in the tape are quite well aligned with only a small Gaussian width $\left(<\sim 10^{\circ}\right)$ to the angular distribution of the ab planes about the tape plane. This means that even for a magnetic field in the tape plane, there will be a c-axis component for some of the grains that will result in a more rapid field dependence. The splayed columnar defects decrease the c-axis field dependence (nearly equivalent to the B ||tape normal dependence) as discussed above. Thus for B||tape normal the critical current of the misoriented grains will also be enhanced, leading to a smaller overall field dependence.

\section{Proton Energy and Activation}

Periodic measurements of the induced activity in the tapes beginning about 60 days after the irradiations and continuing to 250 days were made. The data for 14 tapes irradiated to different doses with $800 \mathrm{MeV}$ protons can be approximately fitted by an exponential decay. However, the calculated half-life increases from the 37 days, when calculated for the data $<120$ days, to 57 days for all the data. This implies that the decay is at least partly nonexponential. Analysis of the decay of daughter products from complex reactions such as fission, which produces a statistical distribution of fission fragment products, predicts that the decay should follow an inverse time dependence at long times. Spallation, which also occurs in the sample and also produces many different daughter products, should also lead to a similar activation - time dependence. The same data were therefore fitted to $1 /\left(\right.$ Time $\left.-t_{0}\right)$. The fits are in most cases fairly accurate and yield $a t_{o}$ of 42 days. The activity of some of the tapes had dropped to only 5-10 times background, making for potentially less accurate measurements for longer times.

There is very little energy dependence to the fission cross section for high-energy protons on $\mathrm{Bi}$ nuclei for energies of $\sim 1000 \mathrm{MeV}$ down to $200 \mathrm{MeV}$, so little change in the defect production and the nature of the defects is expected. The energy released during the fission process $(\sim 200 \mathrm{MeV})$ is independent of the kinetic energy of the incident proton, and is instead characteristic of the fission process itself. 
Activation from a second type of nuclear reaction, known as spallation, which occurs in the HTS core and the Ag sheath, is expected to be much smaller for incident proton energy of $500 \mathrm{MeV}$ compared to $800 \mathrm{MeV}$. Measurements of the activation of a series of tapes 120 days after irradiation to several defect densities using both 500 and $800 \mathrm{MeV}$ protons show a roughly linear dependence on defect density for a given proton energy. They also show a strong dependence on proton energy: the activation is a factor of three smaller for $500 \mathrm{MeV}$ than for $800 \mathrm{MeV}$ protons.

The activity of a stack of nine tapes irradiated simultaneously was measured individually and then as a stack. The measured activity of a stack of tapes increased much more slowly than linearly with the number of tapes. This indicates that there is a significant amount of shielding of the radiation by the outer tapes. This is turn is evidence for a significant fraction of "soft" (low energy) $\beta$ and $\gamma$ radiation being emitted. In a separate experiment, a microscope slide placed over a tape reduced the measured radiation by $50 \%$, again showing the substantial low energy fraction of emitted radiation.

\section{Comparison with Other Results}

Hensel et al. [10] irradiated $\mathrm{Bi}-2223 / \mathrm{Ag}$ tapes with $600 \mathrm{MeV}$ protons to defect densities of $\sim 0.5$ and $\sim 2 \mathrm{~T}$. They saw qualitatively similar enhancements in the magnetic field dependence of $\mathrm{I}_{\mathrm{c}}$ for $\mathrm{B} \|$ tape normal. For the tape irradiated to a $\mathrm{B} \phi$ of $2 \mathrm{~T}$, they saw an improvement of the field dependence with a $1 \mathrm{~h}$ post irradiation anneal at the high temperature of $750^{\circ} \mathrm{C}$ For an anneal for $1 \mathrm{~h}$ at $825^{\circ} \mathrm{C}$, the defects were substantially recrystallized and the performance similar to that of the unirradiated sample.

The work of Schulz et al. [7] using thermal neutron irradiation of uranium-doped Bi-2223 demonstrates comparable levels of $I_{c}$ enhancement in applied magnetic field. Activation levels are also comparable. This technique has the advantage that thermal neutrons sources are more generally available and may be less expensive to use than high energy proton sources. On the other hand, doping of uranium into the Bi-2223 powder requires chemical compatibility and possible changes in the processing of the tapes and the enhanced difficulty of working with (at least mildly) radioactive powders.

\section{SUMMARY AND CONCLUSIONS}

High energy protons induce fission reactions in $\mathrm{Bi}-2223 / \mathrm{Ag}$ sheathed tapes resulting in the production of splayed columnar defects capable of greatly improving the in-field $\mathrm{I}_{\mathrm{c}}$ performance of the tapes for the magnetic field direction parallel to the tape normal. Very high defect densities $(>\sim 1 \mathrm{~T})$ result in degradation of the self field $\mathrm{I}_{\mathrm{c}}$ and eventually weak link behavior at defect densities $>\sim 2 \mathrm{~T}$.

In a post irradiation annealing study, it was found that for nominal defect densities $\mathrm{B}_{\phi}$ greater than $\sim 1 \mathrm{~T}$, the splayed columnar defects produced by high energy proton irradiation cause degradation in $\mathrm{I}_{\mathrm{c}}$ at zero field and weak link behavior in a magnetic field. However, a short, low temperature post irradiation anneal can produce major healing of the weak links and an enhancement in the zero field $\mathrm{I}_{\mathrm{c}}$. For $\mathrm{B}_{\phi}=1.3 \mathrm{~T}$, the enhancement yields excellent field performance out to at least $1 \mathrm{~T}$ applied field at $75 \mathrm{~K}$.

There were only minor differences found among the set of 9 tapes irradiated simultaneously; the first and last tapes in the stack showed very similar $\mathrm{I}_{c}(\mathrm{~B})$ dependencies.

The activation decays with time in roughly an exponential fashion. However, at longer times the data can also be fit to an inverse time dependence. The activation of the tapes is roughly linear with defect density (and therefore, proton fluence), and it is a factor of 3 lower for tapes irradiated with $500 \mathrm{MeV}$ protons compared with that of tapes irradiated at $800 \mathrm{MeV}$. A set of 9 tapes irradiated to a $\mathrm{B}_{\phi}$ of $1 \mathrm{~T}$ with $500 \mathrm{MeV}$ protons showed that stacks of tapes, similar to layers in a coil, have much smaller measured activity compared with simply summing the results of the individual tape activities, indicating substantial self shielding of the activity.

All of the findings here point toward the use of lower proton energy as very favorable for potential commercial use of this technique to enhance the high temperature, high field 
performance of $\mathrm{Bi}-2223 / \mathrm{Ag}$ tapes. The fission cross section is practically flat from 1000 Mev down to $200 \mathrm{MeV}$, suggesting the latter as an optimum value. This would result in much lower activation of the composite tape from parasitic processes, such as spallation reactions. Reducing the proton energy from 800 or $500 \mathrm{MeV}$ to $200 \mathrm{MeV}$ would also yield a large reduction in the potential cost of a facility for irradiating the tapes.

\section{ACKNOWLEDGEMENTS}

Work at the Los Alamos National Laboratory was performed under the auspices of the U. S. Department of Energy, Office of Energy Efficiency and Renewable Energy, as part of the Superconductivity Program for Electric Systems. The authors wish to acknowledge valuable discussions with members of the Wire Development Group led by American Superconductor Corporation and including Argonne National Laboratory, Los Alamos National Laboratory, Oak Ridge National Laboratory, and the University of Wisconsin.

\section{REFERENCES}

1. Thompson, J.R., Sun, Y. R., Kerchner, H.R., Christen, D.K., Sales, B.C., Chakoumakos, B.C., Marwick, A.D., Civale, L., and Thomson, J. O., Appl. Phys. Lett. 60, p.p. 2306-2308 (1992).

2. Hwa, T., LeDoussal, P., Nelson, D. R, and Vinokur, V. M., Phys. Rev. Lett. 71, pp. 3545-3548 (1993).

3. Krusin-Elbaum, L., Thompson, J.R., Wheeler, R., Marwick, A. D., Li, C., Patel, S., Shaw, D. T., Lisowski, P., and Ullmann, J., Appl. Phys. Lett. 64, pp. 3331-3333 (1994)

4. Safar, H., Cho, J. H., Fleshler, S., Maley, M. P., Willis, J. O., Coulter, J. Y., Ullmann, J. L., Lisowski, P. W., Riley, Jr., G. N., Rupich, M. W., Thompson, J. R., and Krusin-Elbaum, L., Appl. Phys. Lett. 67, pp. 130-132 (1995).

5. Willis, J. O., Safar, H., Cho, J. H., Coulter, J. Y., Maley, M. P., Smith, P. A., Phillips, D. S Ullmann, J. L., Riley, Jr., G. N., Rupich, and Fleshler, S., "High Energy Proton Irradiation Induced Pinning Centers in Bi-2212 and Bi-2223 Superconductors," in Advances in Superconductivity VIII, edited by H. Hayakawa and Y. Enomoto, Springer-Verlag, Tokyo, 1996, pp. 509-512.

6. Budhani, R.C., Willis, J. O., Suenaga, M., Maley, M. P., Coulter, J. Y., Safar, H., Ullmann, J. L., and Haldar, P., J. Appl. Phys. 82, pp. 3014-3016 (1997).

7. Schulz, G. W., Klein, C., Weber, H. W., Moss, S., Zeng, R., Dou, S. X., Sawh, R., Ren, Y., Weinstein, R., Appl. Phys. Lett. 73 pp. $3935-7$ (1998).

8. Malozemoff A. P., Carter, W., Gannon, J., Joshi, C., Miles, P., Minot, M., Parker, D., Riley, G., and Thompson, E., Cryogenics 32 ICMC Supplement pp. 478-483 (1992).

9. Becchetti, F. D., Jänecke, J., Lister, P., Kwiatowski, K., Karwowski, H., and Zhou, S., Phys. Rev. C 28, pp. 276-279 (1983).

10. Hensel, B., Marti, F., Grasso, G., Dhallé, M., Flükiger, R., Paschoud, F., and Victoria, M., IEEE Trans. Appl. Supercond. 7, pp. 2030-2033 (1997). 